\title{
Buchbesprechung
}

\section{River Ecosystem Management: Science for Governing Towards a Sustainable Future}

Stephan Schmutz und Jan Sendzimir (Herausgeber), 2018. 571 Seiten, 123 Abbildungen, davon 82 in Farbe. Aquatic Ecology Series, Springer, Cham, Schweiz. Gebunden, Preis: 49,99 €, ISBN 978-3-319-73249-7 (Hardcover), ISBN 9783-319-73250-3 (eBook).

\author{
Buchbesprechung verfasst von: \\ Roland Psenner
}

Universität Innsbruck, Institut für Ökologie, Technikerstrasse 25, 6020 Innsbruck, Österreich, roland.psenner@uibk.ac.at

Das von Univ.-Prof. Dr. Stephan Schmutz (Institut für Hydrobiologie und Gewässermanagement der Universität für Bodenkultur Wien) und Dr. Jan Sendzimir (International Institute for Applied Systems Analysis, Laxenburg) im Springer Verlag herausgegebene Buch „River Ecosystem Management" behandelt auf 571 Seiten in 29 Kapiteln, verfasst von 28 Autorinnen und Autoren, fast sämtliche Aspekte der Fließgewässerökologie. Beginnend von der Geschichte der Beziehung des Menschen zum Wasser spinnt sich der Faden über Flussmorphologie, Hydrologie, Sedimenttransport und Nährstoffdynamik bis zu den Themen Fischerei, Ökotoxikologie, Klimawandel und Ökosystemleistungen. Dabei werden alle wesentlichen Probleme behandelt, die wir an den Flüssen Europas und weltweit beobachten, wie z. B. die Wirkung von Staudämmen, Schwallbetrieb, Begradigung und Unterbrechung des Fließgewässerkontinuums. Das Buch bleibt nicht bei der Schilderung von Problemen stecken, sondern widmet sich auf mehr als 300 Seiten den Restaurierungsmaßnahmen, dem adaptiven Management von Flusseinzugsgebieten, den gesetzlichen Grundlagen für die Bewirtschaftung von Fließgewässern und den Möglichkeiten der Kooperation zwischen Bürgern, NGOs und den zuständigen Ämtern,
Ministerien und Forschungsinstitutionen. Dazu kommen interessante Fallstudien, beispielsweise über die Donau oder die Theiß, und ein Schwerpunkt auf nachhaltige Fischerei in Burkina Faso.

Warum ist das kein „limnologisches“ Buch? Ganz einfach deswegen, weil es sich an eine Vielzahl von Interessierten, Fachleuten und Nutzern wendet, und damit ein Handbuch für alle Fragen zu Funktion, Leistung und Gefährdung von Flüssen darstellt. Außerdem demonstriert es in zahlreichen Modellen und praktischen Beispielen, wie wir in vernünftiger Weise mit unseren Fließgewässern umgehen sollten. Es ist aber mehr als ein Handbuch mit Anweisungen zur Nutzung und zum Schutz von Fließgewässern, es ist auch ein Weckruf: an den Flüssen sind die Hochkulturen entstanden und mit dem Verlust der Fließgewässer wird auch die Menschheit verschwinden.

Muss man das Buch noch bewerben? Ich denke nicht, denn bei der Präsentation durch die beiden Herausgeber Anfang September 2018 an der Universität für Bodenkultur Wien (BOKU) war es bereits mehr als fünfundachtzigtausendmal heruntergeladen worden, für mich die Bestätigung meiner Auffassung, dass wir sehr lange und sehr hart auf ein solches Werk warten mussten. 